\title{
GENERAL SOLUTIONS FOR PLANE EXTENSIBLE ELASTICAE HAVING NONLINEAR STRESS-STRAIN LAWS*
}

\author{
BY \\ STUART ANTMAN \\ Courant Institute of Mathematical Sciences, New York University
}

\begin{abstract}
A general finite deformation solution is obtained for the equilibrium of hydrostatically loaded elasticae whose underformed shapes are circular arcs. The nonlinear stress-strain relations employed give the bending moment and the axial force as derivatives of a strain energy function with respect to suitable strain measures. The representation of the solution involves arbitrary constants of integration which can accommodate any end conditions consistent with equilibrium. Examples are given. The constraint of inextensibility is examined and a perturbation procedure for small extension is developed. In an appendix, the stress-strain laws are derived by an appropriate reduction of the equations for three-dimensional hyperelasticity.
\end{abstract}

1. Introduction. We define a plane extensible elastica to be a three-dimensional hyperelastic body subject to the geometric restrictions:

(i) The undeformed body admits a smooth plane reference curve $C$, the planes normal to which do not intersect within the body.

(ii) Under the deformation the material curve $c$ (which in its initial configuration is denoted $C$ ) remains planar and the material surfaces which initially were planes normal to $C$ become planes normal to $c$ and suffer neither rotation about $c$ nor deformation.

Let $c$ have arc length $s$ and curvature $\kappa$ and let $C$ have arc length $S$ and curvature $K$. We introduce two strain measures, the extension $\delta$ and the bending $\mu$, by the relations

$$
\delta=d s / d S-1, \quad \mu=(1+\delta)_{\kappa}-K .
$$

In the appendix we show that a plane extensible elastica is characterized by the constitutive equations

$$
M=\partial W / \partial \mu, \quad N=\partial W / \partial \delta
$$

where $M$ is the bending moment, $N$ the axial force and $W=W(\mu, \delta, S)$ is a strain energy function. Had we not chosen $\mu$ as our strain measure of bending, but rather employed the more usual $\kappa-K$, then the resulting constitutive relations would be in an equivalent but more complicated form (cf. Tadjbakhsh [1]). The simplicity of (1.2) renders our ensuing work more transparent. To discover the physical significance of $\mu$, we denote the tangent angle to $c$ by $\phi$ and that to $C$ by $\Phi$. Then $d \phi / d s=-\kappa$, $d \Phi / d S=-K$, so that

$$
\mu=-\frac{d}{d \bar{S}}(\phi-\Phi), \quad \text { or } \quad \mu+K=-\frac{d \phi}{d S} .
$$

*Received February 9, 1967. This paper represents results obtained at the Courant Institute of Mathematical Sciences, New York University, with the National Science Foundation, Grant NSF-GP3465 . 
Moreover, it follows from (1.1) that the strain measure $\mu$ has the property that for a uniform extension of a circular arc into another circular arc of different curvature $\mu$ is zero. Because of this insensitivity to pure extension, $\mu$ is an ideal measure for stability investigations.

We impose the restriction on $W$ that (1.2) can be inverted to yield $\mu$ and $\delta$ as functions of $M$ and $N$. This means that

$$
W_{\mu \mu} W_{\delta \delta}-\left(W_{\mu \delta}\right)^{2} \neq 0,
$$

where the subscripts on $W$ denote partial differentiation with respect to the indicated argument. This requirement is analogous to those usually imposed in three-dimensional elasticity (cf. Truesdell and Toupin [2]).

To obtain a theory for inextensible elasticae, we set $\delta=0$, let $N$ be arbitrary (with respect to constitutive relations), and use the constitutive relation $M=W_{\mu}$, where $\mu$ now equals $\kappa-K$. Corresponding to (1.4), we require $W_{\mu \mu} \neq 0$.

The equilibrium equations for bodies such as the plane extensible elastica can be found from a free body diagram (Fig. 1), or they may be derived by integrating the equations of equilibrium for continua across cross-sectional surfaces (cf. A. E. Green [3]). In terms of the arc length $s$ of $c$, they are

$$
\begin{aligned}
d Q / d s-{ }_{\kappa} N-q & =0, \\
d N / d s+{ }^{\alpha} Q & =0, \\
d M / d s-Q & =0,
\end{aligned}
$$

where $Q$ is the shear resultant and $q$ is the normal load. We have assumed there is neither tangential load nor distributed moment applied to the body. The sign convention used is given in Fig. 1. Since $d s / d S=1+\delta$, we can write (1.5) as

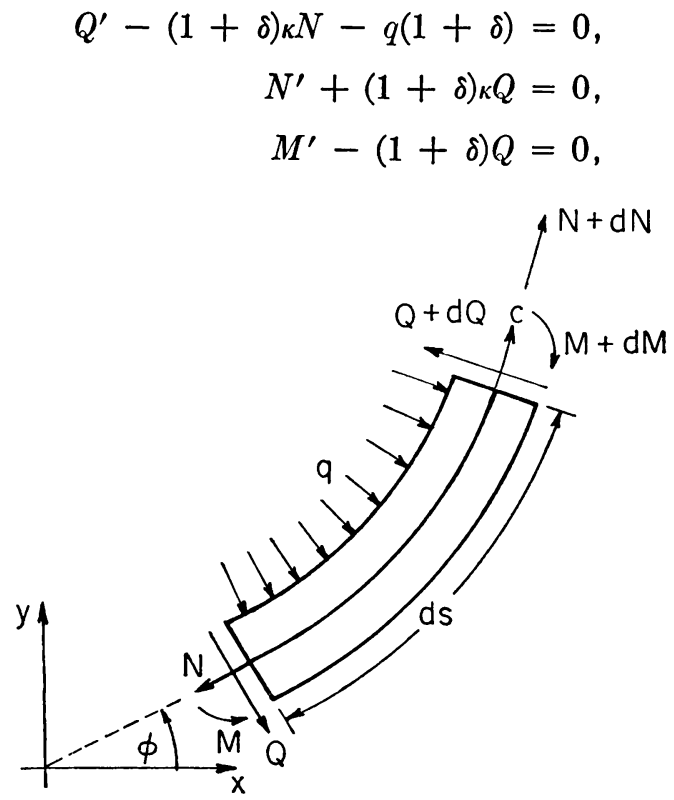

FIG. 1. 
where ${ }^{\prime} \equiv d / d S$. We eliminate $Q$ from (1.6), (1.7), (1.8) and use (1.1) to get

$$
\begin{aligned}
\left(M^{\prime} /(1+\delta)\right)^{\prime}-(\mu+K) N-q(1+\delta) & =0, \\
(\mu+K) M^{\prime}+(1+\delta) N^{\prime} & =0 .
\end{aligned}
$$

Thus we have four equations (1.2), (1.9), and (1.10) for the four unknowns $\mu, \delta$, $M, N$. Once $\mu$ and $\delta$ are found subject to appropriate boundary conditions, (1.1) can be used to find $\kappa$ as a function of $s$; this determines $c$.

2. Integrable cases. Problem 1 . $K$ is constant, $q$ is constant, and the elastica is homogeneous, i.e. $W$ does not depend explicitly on $S$. (These restrictions prevent the appearance of the independent variable $S$ in the governing equations.) This is the most general problem we discuss. We seek a general representation for the solution.

Solution. Multiply the equilibrium equation (1.9) by $M^{\prime} /(1+\delta)$ and use (1.10) to obtain

$$
\frac{1}{2} \frac{d}{d S}\left(\frac{M^{\prime}}{1+\delta}\right)^{2}+\frac{1}{2} \frac{d}{d S} N^{2}-q M^{\prime}=0 .
$$

This equation can be integrated to yield

$$
M^{\prime 2}+(1+\delta)^{2} N^{2}-2 q M(1+\delta)^{2}=a(1+\delta)^{2}
$$

where $a$ is an arbitrary constant of integration. Note that (2.2) is valid for any constitutive hypothesis. The substitution of the stress-strain laws (1.2) into (2.2) yields

$$
W_{\mu}^{\prime 2}+(1+\delta)^{2} W_{\delta}^{2}-2 q(1+\delta)^{2} W_{\mu}=a(1+\delta)^{2},
$$

and the substitution of (1.2) into (1.10) gives

$$
(\mu+K) W_{\mu}^{\prime}+(1+\delta) W_{\delta}^{\prime}=0 .
$$

Since $K$ is a constant, (2.4) can be written as

$$
\left[(\mu+K) W_{\mu}\right]^{\prime}+\left[(1+\delta) W_{\delta}\right]^{\prime}-W_{\mu} \mu^{\prime}-W_{\delta} \delta^{\prime}=0 .
$$

Since $W$ does not depend explicitly on $S$,

$$
W^{\prime}=W_{\mu} \mu^{\prime}+W_{\delta} \delta^{\prime} .
$$

Hence (2.5) may be integrated to yield

$$
(\mu+K) W_{\mu}+(1+\delta) W_{\delta}-W=b,
$$

where $b$ is an arbitrary constant of integration. Equation (2.6) is just an algebraic relation between $\delta$ and $\mu$. We assume that (2.6) can be solved for $\delta=\delta^{*}(\mu)$ or for $\mu=$ $\mu^{*}(\delta)$. Then $(2.3)$ can be written as

$$
S= \pm \int f^{-1 / 2} d W_{\mu}+c,
$$

where $c$ is an arbitrary constant of integration and

$$
f=a(1+\delta)^{2}+2 q(1+\delta)^{2} W_{\mu}-(1+\delta)^{2} W_{\delta}^{2} .
$$

In particular, when (2.6) yields $\delta=\delta^{*}(\mu)$, i.e. when the implicit function condition for $(2.6)$

$$
(\mu+K) W_{\mu \delta}+(1+\delta) W_{\delta \delta} \neq 0
$$


is satisfied, then

$$
\frac{d \delta}{d \mu}=-\frac{(\mu+K) W_{\mu \mu}+\left[1+\delta^{*}(\mu)\right] W_{\delta \mu}}{(\mu+K) W_{\mu \delta}+\left[1+\delta^{*}(\mu)\right] W_{\delta \delta}}
$$

and

$$
d W_{\mu}=\frac{\left[1+\delta^{*}(\mu)\right]\left[W_{\mu \mu} W_{\delta \delta}-W_{\mu \delta}^{2}\right]}{(\mu+K) W_{\mu \delta}+\left[1+\delta^{*}(\mu)\right] W_{\delta \delta}} d \mu
$$

In (2.9) the arguments of the derivatives of $W$ are $\left(\mu, \delta^{*}(\mu)\right)$. Similarly, when we know $\mu=\mu^{*}(\delta)$,

$$
d W_{\mu}=-\frac{(1+\delta)\left[W_{\mu \mu} W_{\delta \delta}-W_{\mu \delta}^{2}\right]}{\left[\mu^{*}(\delta)+K\right] W_{\mu \mu}+(1+\delta) W_{\mu \delta}} d \delta
$$

Here the arguments of the derivatives of $W$ are $\left(\mu^{*}(\delta), \delta\right)$.

Note that by virtue of (1.4), the numerators of (2.9) and (2.10) do not vanish. Thus (2.6) and (2.7) implicitly give the general solution $\mu(S), \delta(S)$ of Problem 1 in terms of three arbitrary constants $a, b, c$. To find the shape of the elastica, we introduce Cartesian coordinates $x$ and $y$ by the relations

$$
d x / d s=\cos \phi, \quad d y / d s=\sin \phi,
$$

where $\phi$ is the tangent angle to the curve. Equations (2.11) and (1.3) imply

$$
\begin{aligned}
& \phi(S)-\phi\left(S_{0}\right)=-\int_{S_{0}}^{S}\left[\mu\left(S_{1}\right)+K\right] d S_{1}, \\
& x(S)-x\left(S_{0}\right)=\int_{S_{0}}^{S}\left[1+\delta\left(S_{1}\right)\right] \cos \phi\left(S_{1}\right) d S_{1}, \\
& y(S)-y\left(S_{0}\right)=\int_{S_{0}}^{S}\left[1+\delta\left(S_{1}\right)\right] \sin \phi\left(S_{1}\right) d S_{1},
\end{aligned}
$$

where $\phi\left(S_{0}\right), x\left(S_{0}\right), y\left(S_{0}\right)$ are arbitrary integration constants and $S_{0}$ is any convenient value of $S$.

Thus our sixth-order system of ordinary differential equations, (1.9), (1.10), (1.3), (2.11) has a general representation for its solution given by (2.6), (2.7), and (2.12). This representation involves the six arbitrary integration constants $a, b, c, \phi\left(S_{0}\right), x\left(S_{0}\right)$, $y\left(S_{0}\right)$, which accommodate any set of boundary conditions consistent with equilibrium. The constants are then determined by the solution of transcendental equations involving functions generated by the quadratures (2.6), (2.7), (2.12). ${ }^{1}$ (If an end of the rod is fixed, then the constants $x\left(S_{0}\right)$ and $y\left(S_{0}\right)$ merely locate the origin of coordinates and may be assigned in any convenient way. The constant $\phi\left(S_{0}\right)$ plays an analogous role if an end has a fixed slope.)

Such representations seem to afford tractable numerical approaches to a number of problems of stability. Moreover, an equation such as (2.7) is a fruitful source of qualitative information on the nature of solutions.

1The question of the existence and number of solutions of these transcendental equations depends on the nature of $W$ and will be considered in subsequent work. We tacitly assume here that it is meaningful to talk about solutions. 
Example. $W=(1 / 2) E I \mu^{2}+(1 / 2) E A \delta^{2} . E I$ and $E A$ are constants. $(E$ is Young's modulus, $A$ is the cross-sectional area, and $I$ is the moment of inertia of the crosssection about the axis that is normal to the plane of bending and passes through the centroid of the rod.) For this especially simple form of $W$, we have $M=E I \mu$ and $N=E A \delta$.

Since $1+\delta>0,(2.6)$ can be written in the form

$$
1+\delta=\left[B-(I / A)(\mu+K)^{2}\right]^{1 / 2}
$$

where $B$ is an arbitrary constant. Then

$f=\left[B-(I / A)(\mu+K)^{2}\right]\left\{a+2 E I q \mu-(E A)^{2}\left[\left(B-(I / A)(\mu+K)^{2}\right)^{1 / 2}-1\right]^{2}\right\}$

and $d W_{\mu}=E I d \mu$. The change of variables $\mu+K=(A B / I) \sin \theta, w=\tan \theta / 2$ reduces (2.7) to an elliptic integral in Weierstrass form. For the solution of a given boundary value problem, however, it is doubtful that the use of Weierstrass elliptic functions would be more expeditious than either the use of Jacobi elliptic functions or the direct numerical integration of (2.7) with $f$ given by (2.14).

To indicate the structure of a typical boundary value problem, we examine the concrete example of a hinged circular arch of angle $2 \alpha$ subject to a hydrostatic pressure (Fig. 2). The boundary conditions are

$$
\begin{gathered}
M( \pm \alpha / K)=E I \mu( \pm \alpha / K)=0, \\
x(\alpha / K)-x(-\alpha / K)=(2 / K) \sin \alpha, \\
y(\alpha / K)-y(-\alpha / K)=0 .
\end{gathered}
$$

We introduce the new variables

$$
\zeta=\alpha(\mu+K) / K, \quad S=\alpha t / K, \quad \beta^{2}=K^{2} I / \alpha^{2} A, \quad p=\alpha^{3} q / K^{3} E I .
$$

Equations (1.9), (2.3), and (2.4) then become

$$
\begin{gathered}
\frac{d}{d t}\left[(1+\delta)^{-1} \frac{d \zeta}{d t}\right]-\frac{1}{\beta^{2}} \zeta \delta-p(1+\delta)=0 \\
\beta^{2} \zeta \frac{d \zeta}{d t}+(1+\delta) \frac{d \delta}{d t}=0 \\
\left(\frac{d \zeta}{d t}\right)^{2}=(1+\delta)^{2}\left[a_{0}+2 p(\zeta-\alpha)-\frac{1}{\beta^{4}} \delta^{2}\right],
\end{gathered}
$$
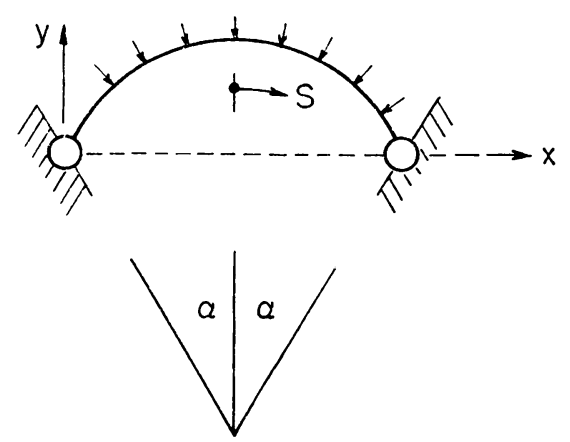

FIG. 2. 
and (2.7), (2.13), (2.12), (2.14), (2.15) reduce to the following system for $\zeta(t)$ :

$$
\begin{gathered}
\pm(t+1)=\int_{\alpha}^{\zeta(t)} h(\zeta)^{-1 / 2} d \zeta, \\
1+\delta=\left(\gamma^{2}-\beta^{2} \zeta^{2}\right)^{1 / 2}, \\
\phi(t)-\phi(-1)=-\int_{-1}^{t} \zeta(\tau) d \tau, \\
\zeta(1)=\alpha, \\
\int_{-1}^{1}[1+\delta(t)] \cos \phi(t) d t=\frac{2 \sin \alpha}{\alpha}, \\
\int_{-1}^{1}[1+\delta(t)] \sin \phi(t) d t=0,
\end{gathered}
$$

where

$$
h(\zeta)=\left(\gamma^{2}-\beta^{2} \zeta^{2}\right)\left\{a_{0}+2 p(\zeta-\alpha)-\frac{1}{\beta^{4}}\left[\left(\gamma^{2}-\beta^{2} \zeta^{2}\right)^{1 / 2}-1\right]^{2}\right\} .
$$

Here $a_{0}, \gamma^{2}$, and $\phi(-1)$ are constants to be evaluated by the subsidiary conditions (2.23), (2.24), (2.25). Equation (2.19) is an integral of (2.17) and (2.18). Equation (2.20) is the integral of (2.19) subject to the boundary condition $\zeta(-1)=\alpha$ and (2.21) is the integral of (2.18). In (2.20), the upper limit $\zeta(t)$ is presumed to be in an interval of the $\zeta$-axis containing $\zeta=\alpha$ for which there is a single-valued representation for $t$ as a function of $\zeta$. Equation (2.21) and the boundary conditions $\zeta( \pm 1)=\alpha$ imply that the extension at each end of the rod is the same. Denoting this extension by $\delta_{0}$, we observe that

$$
\gamma^{2}=\left(1+\delta_{0}\right)^{2}+\beta^{2} \alpha^{2} .
$$

Since the load is hydrostatic, the vertical reactions at each end of the arch can be found to be $(q \sin \alpha) / K$. From Fig. 1, we then obtain the conditions

$$
N( \pm 1) \sin \phi( \pm 1)+Q( \pm 1) \cos \phi( \pm 1)= \pm \frac{q \sin \alpha}{K} .
$$

Using the equilibrium equation (1.7), the constitutive equation $N=E A \delta$, and the change of variables (2.16), we ultimately obtain the alternate set of subsidiary conditions:

$$
\begin{aligned}
\left(\beta^{4} a_{0}-\delta_{0}^{2}\right) \cos ^{2} \phi(-1) & =\left[\beta^{2} p \frac{\sin \alpha}{\alpha}+\delta_{0} \sin \phi(-1)\right]^{2} ; \\
\left(\beta^{4} a_{0}-\delta_{0}^{2} x\right) \cos ^{2} \phi(1) & =\left[\beta^{2} p \frac{\sin \alpha}{\alpha}-\delta_{0} \sin \phi(1)\right]^{2} .
\end{aligned}
$$

The original differential equations (2.17) and (2.18) admit constant solutions. But by means of these equations one can easily show that the constant solution $\zeta=\alpha$ satisfying the boundary conditions $\zeta( \pm 1)=\alpha$, is trivial because it can satisfy the remaining conditions (2.24) and (2.25) only if $\kappa=K, \delta=0$, and $q=0$. (For some other systems of boundary conditions, however, there are nontrivial constant solutions.)

We now examine the restrictions that the boundary conditions $\zeta( \pm 1)=\alpha$ impose 
on the nature of solutions of our system by studying $h(\zeta)$. From (2.26) we have that

$$
h(\alpha)=\left(1+\delta_{0}\right)^{2}\left(a_{0}-\delta_{0}^{2} / \beta^{4}\right) .
$$

Equation (2.19) or (2.29) then implies that $h(\alpha)>0$ for a nontrivial solution. Equation (2.26) also shows that $h(\zeta)$ has zeros at $\zeta= \pm \gamma / \beta$. Now since $\zeta$ must have the same value $\alpha$ at each end of the rod, Rolle's theorem implies that there is a value $t=T$ in the open interval $(-1,1)$ for which $d \zeta / d t=0$ but $d^{2} \zeta / d t^{2} \neq 0$. If the requirement $1+\delta>0$ is not to be violated, these conditions imply that $h(\zeta)$ has a simple zero at $\zeta(T)$ with $|\zeta(T)|<\gamma / \beta$. If we let $\zeta_{1}$ be the greatest zero of $h(\zeta)$ less than $\alpha$ at which $h(\zeta)$ changes sign and $\zeta_{2}$ be the least zero of $h(\zeta)$ greater than $\alpha$ at which $h(\zeta)$ changes sign, then at least one of the zeros $\zeta_{1}$ and $\zeta_{2}$ lies in the open interval $(-\gamma / \beta, \gamma / \beta)$ of the $\zeta$-axis and $h(\zeta)$ has the form shown in Fig. 3.

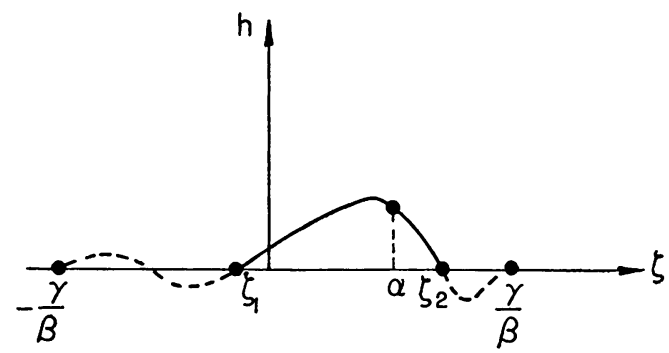

FIG. 3.

When $h(\zeta)$ has this form the qualitative nature of the solution function $\zeta(t)$ given by (2.19) is well known (cf. Synge and Griffith [4]). In particular, $\zeta(t)$ is periodic with half-period $\omega=\int_{\zeta_{2}}^{\zeta_{2}} h(\zeta)^{-1 / 2} d \zeta$, is bounded in the strip $\zeta_{1} \leq \zeta(t) \leq \zeta_{2}$, and is symmetric about its points of tangency to the lines $\zeta=\zeta_{1}$ and $\zeta=\zeta_{2}$. These properties are illustrated in Fig. 4.

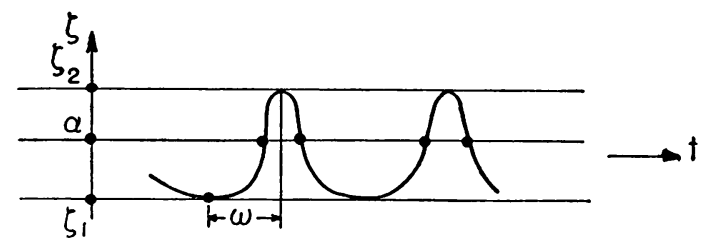

FIG. 4.

We therefore deduce that for each value of $n, n=0,1,2, \cdots$, there can be one symmetric solution with $2 /(2 n+1) \leq \omega<1 / n$, one symmetric solution with $1 /(n+1)<$ $\omega \leq 2 /(2 n+1)$, and two asymmetric solutions (mirror images of each other) with $\omega=1 /(n+1)$, each such solution satisfying the boundary conditions $\zeta( \pm 1)=\alpha$. If one of the zeros $\zeta_{1}$ and $\zeta_{2}$, say $\zeta_{2}$, should have absolute value greater than $\gamma / \beta$, then any solution $\zeta=\zeta(t)$ that touches the line $\zeta=\zeta_{2}$ must be discarded because it violates the restriction that $1+\delta>0$. (We have already shown that both zeros cannot simultaneously exceed $\gamma / \beta$ in absolute value.) The additional subsidiary conditions (2.24) and (2.25) determine the shape and size of the solution curve in Fig. 4 and restrict the values of parameters for which such solutions may arise. 
Problem 2. To the specifications of Problem 1 we add the restriction that the elastica is inextensible.

Solution. Substitute the constitutive relation $M I=W_{\mu}$ into (2.2) and (1.10) to get

$$
\begin{aligned}
W_{\mu}^{\prime 2}+N^{2}-2 q W_{\mu} & =a, \\
(\mu+K) W_{\mu}^{\prime}+N^{\prime} & =0 .
\end{aligned}
$$

Since $K$ is constant and the elastica is homogeneous, (2.32) can be integrated to yield the solution for $N$ :

$$
N+(\mu+K) W_{\mu}-W=b .
$$

When this is substituted into (2.31), the latter can be written in the form

$$
S= \pm \int f(\mu)^{-1 / 2} W_{\mu \mu} d \mu
$$

where

$$
f(\mu)=a+2 q W_{\mu}-\left[b-(\mu+K) W_{\mu}+W\right]^{2} .
$$

When $W$ is in the classical form, $W=(1 / 2) E I \mu^{2}$, then

$$
f(\mu)=a+2 E I q \mu-\left[b-E I \mu(\mu+K)+\frac{1}{2} E I \mu^{2}\right]^{2} .
$$

Equation (2.34) then gives $\mu$ as a function of $S$ in terms of Weierstrass or Jacobi elliptic functions. The analysis of the qualitative behavior of solutions is simplified by the absence of the thickness parameter $\beta$ that was used in the example of Problem 1 .

Problem 3. To the specifications of Problem 1 we add the restriction that $q=0$. This condition enables us to find the resultants $N$ and $Q$ as functions of the tangent angle $\phi$ from a free body diagram.

Solution. We introduce a spatial Cartesian coordinate system $(x, y)$ in the plane of bending and measure $\phi$ in a counter-clockwise sense (cf. Fig. 5).

Let $P_{1}$ and $P_{2}$ be the components of the force and $M_{0}$ the applied moment at one end of the elastica. Since $q=0$, the force and moment are known at the other end. (If the relative displacements or angles rather than the forces or moments were prescribed at the ends, then $P_{1}, P_{2}$ or $M_{0}$ would be unknowns, which could be expressed in terms of the given displacements or angles by means of the solution.)

From a free-body diagram we obtain

$$
\begin{aligned}
& Q=-P_{2} \cos \phi+P_{1} \sin \phi, \\
& N=-\left(P_{1} \cos \phi+P_{2} \sin \phi\right) .
\end{aligned}
$$

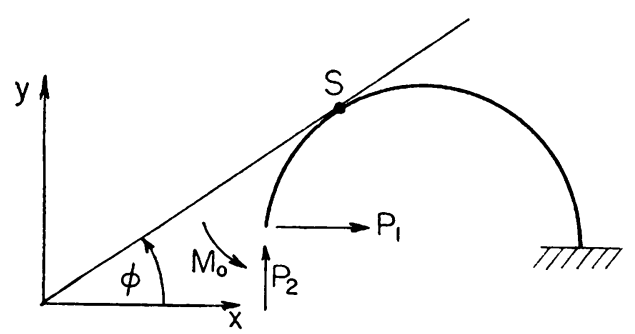

Fia. 5. 
Equation (2.6) and the relation $N=W_{\delta}$ yield the governing equations for this problem:

$$
\begin{gathered}
(\mu+K) W_{\mu}+(1+\delta) W_{\delta}-W=b, \\
W_{\delta}=-\left(P_{1} \cos \phi+P_{2} \sin \phi\right) .
\end{gathered}
$$

We recall that

$$
\mu+K=-\phi^{\prime} .
$$

Equation (2.37) is just an implicit algebraic relation between $\mu$ and $\delta$. To solve (2.37) for $\delta=\delta^{*}(\mu)$, we must have the following implicit function condition satisfied:

$$
(K+\mu) W_{\mu \delta}+(1+\delta) W_{\delta \delta} \neq 0 .
$$

If this condition is not met we must revert to the more general formulation of Problem 1 . If (2.40) is satisfied then the left-hand side of $(2.38)$ can be written as $W_{\delta}\left(\mu, \delta^{*}(\mu)\right)$ and the condition that (2.38) be solvable for $\mu$ in terms of $\phi$ then reduces to

$$
(K+\mu)\left(W_{\mu \mu} W_{\delta \delta}-W_{\mu \delta}^{2}\right) \neq 0 .
$$

By virtue of (1.4), this is valid except where the curvature $\kappa \equiv(K+\mu) /(1+\delta)$ vanishes. At such inflection points solutions can be pieced together (cf. Love [5]). Thus (2.38) can be written in the form

$$
\phi^{\prime} \equiv-(\mu+K)=F\left(P_{1} \cos \phi+P_{2} \sin \phi\right)
$$

so that $\phi$ is representable by the quadrature

$$
S=\int \frac{d \phi}{F}
$$

When $W=(1 / 2) E I \mu^{2}+(1 / 2) E A \delta^{2},(2.37)$ reduces to (2.13) and the function $F$ defined in (2.42) has form

$$
(A / I)^{1 / 2}\left\{B-\left[1-\left(P_{1} \cos \phi+P_{2} \sin \phi\right) / E A\right]^{2}\right\}^{1 / 2}
$$

Equation (2.43) then leads to a representation for $\phi$ directly in terms of Jacobian elliptic functions. For this choice of $W$, the solution for an initially straight two hinged bar is given by Pflüger [6].

Problem 4. We add to the specifications of Problem 3 the restriction that the elastica be inextensible.

Solution. Equations (2.33) and (2.36) are still valid and can be combined to yield

$$
(\mu+K) W_{\mu}-W=b+P_{1} \cos \phi+P_{2} \sin \phi .
$$

This can be solved for $\mu$ as a function of $\phi$ if

$$
(\mu+K) W_{\mu \mu} \neq 0 .
$$

Since we require $W_{\mu \mu} \neq 0,(2.46)$ fails only at inflection points.

For $W=(1 / 2) E I \mu^{2}$, the solution can be represented in terms of Jacobi elliptic functions. The solution for initially straight hinged elasticae is given by Love [5] and a compendium of other solutions is given by Frisch-Fay [7].

3. Perturbation theory for small extensions. In many elastica problems of practical interest the extension is small and the solution to the inextensible problem closely approximates experimental results. To exploit the relative simplicity of the form of 
solution for the inextensible elastica, we now formulate a perturbation technique to treat the case of small extension. ${ }^{2}$ Our formalism is somewhat more direct than that used by Spencer [8] for the treatment of small compressibility in three dimensional theory.

Let $\epsilon$ be a small dimensionless parameter characterizing the extension. Let

$$
\delta=\epsilon e .
$$

Assume that $\mu, e, M, N$ depend smoothly on $\epsilon$ :

$$
\mu=\mu(\epsilon), \quad e=e(\epsilon), \quad M=M(\epsilon), \quad N=N(\epsilon) .
$$

Here we suppress explicit dependence on $S$. Assume that $W$ can be written in the form

$$
W(\mu, \delta)=U(\mu)+\epsilon V(\mu, e),
$$

where $U$ and $V$ are smooth functions of their arguments in a neighborhood of $\mu(0), e(0)$. Then

$$
\begin{gathered}
M=W_{\mu}=U_{\mu}(\mu)+\epsilon V_{\mu}(\mu, e), \\
N=W_{\delta}=V_{\epsilon}(\mu, e) .
\end{gathered}
$$

By repeatedly differentiating (3.4) and (3.5) with respect to $\epsilon$ and then setting $\epsilon=0$, we obtain the sequence of equations

$$
\begin{aligned}
M(0) & =U_{\mu}(\mu(0)), \\
N(0) & =V_{\epsilon}(\mu(0), e(0)), \\
M_{\epsilon}(0) & =U_{\mu \mu}(\mu(0)) \mu_{\epsilon}(0)+V_{\mu}(\mu(0), e(0)), \\
N_{\epsilon}(0) & =V_{e \mu}(\mu(0), e(0)) \mu_{\epsilon}(0)+V_{e \epsilon}(\mu(0), e(0)) e_{\epsilon}(0),
\end{aligned}
$$

Doing the same with the equilibrium equations (1.9) and (1.10), we obtain

$$
\begin{gathered}
M^{\prime \prime}(0)-(K+\mu(0)) N(0)-q=0, \\
(K+\mu(0)) M^{\prime}(0)+N^{\prime}(0)=0, \\
M_{\epsilon}^{\prime \prime}(0)-(K+\mu(0)) N_{\epsilon}(0)-\mu_{\epsilon}(0) N(0)-q c(0)=M^{\prime \prime}(0) e(0)+M^{\prime}(0) e^{\prime}(0), \\
(K+\mu(0)) M_{\epsilon}^{\prime}(0)+\mu_{\epsilon}(0) M^{\prime}(0)+N_{\epsilon}^{\prime}(0)=-N^{\prime}(0) c(0) .
\end{gathered}
$$

Equations (3.6), (3.10), (3.11) are just the field equations for the inextensional theory in terms of the three unknowns $M(0), N(0), \mu(0)$. When these equations are solved, we can find $e(0)$ from (3.7). This process can be continued: (3.8), (3.12), and (3.13) can be solved for $M_{\epsilon}(0) . N_{\epsilon}(0)$. and $\mu_{\epsilon}(0)$. Then $e_{\epsilon}(0)$ can be found from (3.9). The final representations are of the form $\mu=\mu(0)+\mu_{\epsilon}(0) \epsilon+(1 / 2) \mu_{\epsilon \epsilon}(0) \epsilon^{2}+\cdots$, etc.

Example. $W=(1 / 2) E I \mu^{2}+(1 / 2) E A \delta^{2}$. Then $U=(1 / 2) E I \mu^{2}, V=(1 / 2) \epsilon E A e^{2}$. Equation (3.7) then implies $\epsilon e(0)=N(0) / E A$, a physically reasonable result.

Appendix: Derivation of constitutive laws. We now derive the stress-strain laws for

${ }^{2}$ The method used here was proposed by Professor J. B. Keller. 
elasticae by employing our definition of an elastica as a certain three-dimensional body and specializing the three-dimensional stress-strain laws to this model. ${ }^{3}$

We begin by obtaining an analytic characterization for the definition of a plane extensible elastica given in Sec. 1 .

Let $\pi$ be the plane to which $c$ is restricted. Let $X^{1}=X, X^{2}=Y$, and $X^{3}=S$ be convected coordinates assigned in the undeformed body with $S$ measuring arc length of $C, X$ measuring distance of points from $\pi$, and $Y$ measuring distance of points from the cylinder generated by normals to $\pi$ through $C$. Let $\mathbf{r}^{*}$ be the position vector to any point on $c$. Let $a_{1}$ be a constant unit vector normal to $\pi, a_{2}(S)$ be the unit vector normal to $c$ lying in $\pi$ and let $\mathrm{a}_{3}(S) \equiv d \mathrm{r}^{*} / d S$ be the vector tangent to $c$ whose magnitude in the undeformed configuration is unity. $\mathbf{a}_{1}, \mathbf{a}_{2}$ and $\mathbf{a}_{3}$ are to form a right-handed system (cf. Fig. 6).

The restrictions (i) and (ii) defining an elastica can now be incorporated in the following representation for the position vector $\mathbf{r}$ to any point in the deformed body:

$$
\mathbf{r}=\mathbf{r}^{*}+X \mathbf{a}_{1}+Y \mathbf{a}_{2} .
$$

(For an unconstrained rod, $\mathbf{r}$ would have a formal representation as an infinite series in $X$ and $Y$ with no such restrictions on the $\mathbf{a}_{i}$. Cf. Antman and Warner [9], A. E. Green [3]).

We introduce the base vectors $\mathbf{g}_{i}$ for the deformed configuration by the relations

$$
\mathbf{g}_{1}=\frac{\partial \mathbf{r}}{\partial X}=\mathbf{a}_{1}, \quad \mathbf{g}_{2}=\frac{\partial \mathbf{r}}{\partial Y}=\mathbf{a}_{2}, \quad \mathbf{g}_{3}=\frac{\partial \mathbf{r}}{\partial S}=\mathbf{a}_{3}+\kappa Y \mathbf{a}_{3},
$$

where $\kappa$ is the curvature of the deformed rod. The material strain tensor $E_{K L}$ has the form

$$
2 E_{K L}=\mathbf{g}_{K} \cdot \mathbf{g}_{L}-\mathbf{G}_{K} \cdot \mathbf{G}_{L}=2 \delta_{K}^{3} \delta_{L}^{3}\left[E_{(0)}+E_{(1)} Y+E_{(2)} Y^{2}\right],
$$

where $\mathbf{G}_{K}$ is the base vector for the undeformed configuration and where

$$
2 E_{(0)}=a_{3} \cdot a_{3}-1=a_{33}-1, \quad 2 E_{(1)}=2\left(a_{33} \kappa-K\right), \quad 2 E_{(2)}=\kappa^{2} a_{33}-K^{2} .
$$

Here $K$ is the curvature of $C$. Note that $d s / d S=\left(a_{33}\right)^{1 / 2}=1+\delta$.

We define the stress resultant vector $\mathbf{n}$ and the couple resultant vector $\mathbf{m}$ :

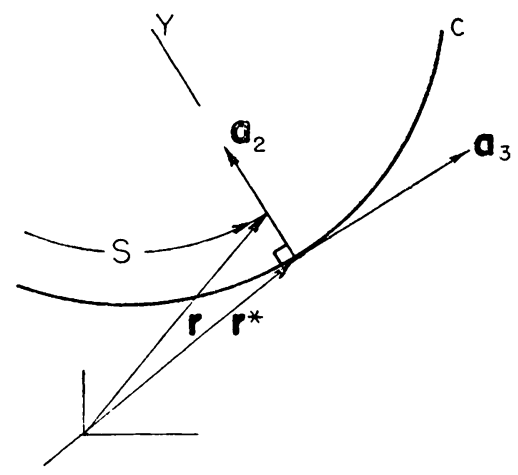

FIG. 6.

${ }^{3}$ An alternative variational approach in which the elastica was treated as a one-dimensional body was carried out by Tadjbakhsh [1]. Equivalent constitutive relations were obtained. 


$$
\mathbf{n}=\int_{A} g^{1 / 2} \mathbf{t}^{3} d X d Y, \quad \mathbf{m}=\int_{A}\left(\mathbf{r}-\mathbf{r}^{*}\right) \times g^{1 / 2} \mathbf{t}^{3} d X d Y
$$

where $g=\operatorname{det}\left(\mathbf{g}_{K} \cdot \mathbf{g}_{L}\right), A$ is the cross-sectional area and $\mathbf{t}^{3}=t^{3 i} \mathbf{g}_{i}$ where $t^{i j}$ is the stress tensor and the summation convention holds (cf. A. E. Green [3]).

In convected coordinates, the stress-strain laws for unconstrained hyperelastic bodies can be written in the form

$$
g^{1 / 2} t^{k l}=G^{1 / 2} \partial \Sigma / \partial E_{k l}
$$

where $G$ is the value of $g$ in the undeformed configuration and $\Sigma$ is the strain energy function. For the elastica with constraints characterized by (A.1) or (A.3), the stressstrain laws become

$$
g^{1 / 2} t^{33}=G^{1 / 2} \partial \Sigma / \partial E_{33}
$$

and all other $t^{i j}$ are arbitrary (cf. Truesdell and Toupin [10] or Ericksen and Rivlin [11]).

From (A.3) we observe (Naghdi and Nordgren [12])

$$
\partial \Sigma / \partial E_{(n)}=Y^{n} \partial \Sigma / \partial E_{33}, \quad n=0,1,2 .
$$

We define the strain energy per unit length of $C$ by

$$
W \equiv \int_{A} G^{1 / 2} \Sigma d X d Y
$$

Then the physical component of $\mathbf{n}$ in the direction $\mathrm{a}_{3}$, which we call the axial force $N$, is given by

$$
\begin{aligned}
N \equiv \mathbf{n} \cdot \frac{\mathbf{a}_{3}}{\left(a_{33}\right)^{1 / 2}} & =\int_{A} g^{1 / 2} t^{33} \mathbf{g}_{i} \cdot \frac{\mathbf{a}_{3}}{\left(a_{33}\right)^{1 / 2}} d X d Y \\
& =\int_{A} g^{1 / 2} t^{33}\left(a_{33}\right)^{1 / 2}(1+\kappa Y) d X d Y \\
& =\left(a_{33}\right)^{1 / 2} \int_{A} G^{1 / 2} \frac{\partial \Sigma}{\partial E_{33}^{1}}(1+\kappa Y) d X d Y \\
& =\left(a_{33}\right)^{1 / 2}\left[\frac{\partial W}{\partial E_{(0)}}+\kappa \frac{\partial W}{\partial E_{(1)}^{\prime}}\right]
\end{aligned}
$$

where we have used (A.5), (A.2), (A.6), (A.7) and (A.8). Similarly the bending moment $M \equiv \mathrm{m} \cdot \mathrm{a}_{1}$ has the form

$$
M=\left(a_{33}\right)^{1 / 2}\left[\partial W / \partial E_{(1)}+\kappa \partial W / \partial E_{(2)}\right] .
$$

Other stress resultants are arbitrary as a result of (A.6).

We can put the constitutive relations (A.9) and (A.10) into a far more elegant form by introducing the two strain measures of (1.1). From (A.4) we then have

$$
E_{(0)}=\frac{1}{2}\left[(1+\delta)^{2}-1\right], \quad E_{(1)}=(1+\delta)(\mu+K)-K, \quad E_{(2)}=\frac{1}{2}\left[(\mu+K)^{2}-K^{2}\right] .
$$

By the chain rule of partial differentiation, we have 


$$
\begin{aligned}
& \frac{\partial W}{\partial \delta}=\frac{\partial W}{\partial E_{(0)}}(1+\delta)+\frac{\partial W}{\partial E_{(1)}}(\mu+K) \\
& \frac{\partial W}{\partial \mu}=\frac{\partial W}{\partial E_{(1)}}(1+\delta)+\frac{\partial W}{\partial E_{(2)}}(\mu+K)
\end{aligned}
$$

Comparing these results with (A.9) and (A.10) we obtain

$$
M=\frac{\partial W}{\partial \mu}, \quad N=\frac{\partial W}{\partial \delta} .
$$

Acknowledgement. The author is gratefully indebted to Professor Joseph B. Keller for his helpful criticism of this work.

\section{BIBLIOGRAPHY}

1. I. Tadjbakhsh, The variational theory of the plane motion of the extensible elastica, Int. J. Eng. Sci. 4, 433-450 (1966)

2. C. Truesdell and R. Toupin, Static grounds for inequalities in finile strain of elastic materials, Arch. Rational Mech. Anal. 12, 1-33 (1963)

3. A. E. Green, The equilibrium of rods, Arch. Rational Mech. Anal. 3, 417-421 (1959)

4. J. L. Synge and B. A. Griffith, Principles of mechanics, 3rd ed., McGraw-Hill, New York, 1959

5. A. E. H. Love, $A$ treatise on the mathematical theory of elasticity, 4th ed., Dover, New York, 1944

6. A. Pflüger, Stabilitätsprobleme der Elastostatik, Springer-Verlag, Berlin, 1964

7. R. Frisch-Fay, Flexible bars, Butterworths, Washington, 1962

8. A. J. M. Spencer, Finite deformation of an almost incompressible elastic solid, I.U.T.A.M. International Symposium, Haifa, Israel, 1962; Second order effects in elasticity, plasticity, and fluid dynamics, edited by M. Reiner and D. Abir, Macmillan, New York, 1964

9. S. Antman and W. Warner, Dynamical theory of hyperelastic rods, Arch. Rational Mech. Anal. 23, 135-162 (1966)

10. C. Truesdell and R. Toupin, The classical field theories, Handbuch der Physik, III/1, Springer-Verlag, Berlin, 1960

11. J. L. Ericksen and R. S. Rivlin, Large elastic deformations of homogeneous anisotropic materials, J. Rational Mech. Anal. 3, 281-301 (1954)

12. P. M. Naghdi and R. P. Nordgren, On the nonlinear theory of elastic shells under the Kirchhoff hypothesis, Quart. Appl. Math. 21, 49-59 (1963) 